\title{
From Quantum Dots to Micro Crystals: Organolead Triiodide Perovskite Crystal Growth from Isopropanol Solution
}

\author{
M. B. Johansson, ${ }^{\mathrm{a}, *, \mathrm{z}}$ T. Edvinsson, ${ }^{\mathrm{b}}$ S. Bitter, ${ }^{\text {a }}$ A. I. K. Eriksson, ${ }^{\mathrm{a}}$ E. M. J. Johansson, ${ }^{\mathrm{a}}$ \\ M. Göthelid, ${ }^{c}$ and G. Boschloo ${ }^{\mathrm{a}}$ \\ ${ }^{a}$ Department of Chemistry-the Ångström Laboratory, Div. Physical Chemistry, Uppsala University, SE-751 20 \\ Uppsala, Sweden \\ ${ }^{b}$ Department of Engineering Sciences, Div. Solid State Physics, Uppsala University, SE-751 21 Uppsala, Sweden \\ ${ }^{c}$ KTH-Royal Institute of Technology, Div. Materials and Nano Physics, SE-164 40 Stockholm, Sweden
}

\begin{abstract}
The growth mechanism and dependence on precursor conditions are vital for creation of high quality crystalline materials in many fields. Here the growth from nano sized quantum dots to micro crystalline methyl ammonium lead tri-iodide $\left(\mathrm{MAPbI}_{3}\right)$ perovskites prepared from isopropanol solution are reported. Isopropanol is more environmental friendly compared to the commonly used solvents DMF or DMSO, both with relatively high toxicity and the proposed method can be a useful new route to prepare hybrid perovskites. Three different molar ratios of $\mathrm{MAPbI}_{3}$ perovskite solution (MAI:PbI 2 of 1:1, 2:1 and 0.5:1) were applied to give insights in the crystal formation mechanism also under non-stoichiometric conditions. Perovskite crystal growth is followed by TEM. From XRD powder diffraction the lattice constants have been determined and compared with results from electron diffraction (ED). Interestingly, there seems to be an occurrence of the cubic phase besides the common tetragonal phase at room temperature.
\end{abstract}

Solar cells based on a perovskite-absorber, such as $\mathrm{CH}_{3} \mathrm{NH}_{3} \mathrm{PbI}_{3}$, $\left(\mathrm{MAPbI}_{3}\right)$ have lately become a research area with significant interest due to their high power conversion efficiency (PCE). With novel device architecture, perovskite solar cells have now surpassed $22 \%$ confirmed PCE. ${ }^{1}$ A clue to the high performance of the $\mathrm{MAPbI}_{3}$ material is the reasonably long diffusion length of charge carriers and lack of deep trap states, which depends on the crystallinity and morphology. However, it is still difficult to obtain reproducible performance, which address questions related to the details of the crystal growth chemistry in these materials. The organic-inorganic hybrid $\mathrm{MAPbI}_{3}$ system displays good mobility for both electrons and holes, relatively high stability and tuneable bandgap. ${ }^{2,3}$ The perovskite contains a semiconducting inorganic part $\left(\mathrm{PbI}_{3}{ }^{-}\right)$with an organic counterion $\left(\mathrm{MA}^{+}\right)$formed from the lead iodide $\left(\mathrm{PbI}_{2}\right)$ and methylammonium iodide (MAI) precursors where combined organic-inorganic systems have evolved to many important materials in nature, like nacre and the bone structure, in animals and humans. ${ }^{4}$ The direct structure formation in a low dimensional system has several different shapes such as rectangular and triangular quantum dots..$^{5}$ Thereby, a change in size of the low dimensional nucleating $\mathrm{MAPbI}_{3}$ crystals is likely to change the number of grain boundaries and the orientation between different crystalline domains and thus affect the optoelectronic properties of the material, which also affects the photovoltage and photocurrent in the final device. The reaction processes for the formation of the perovskite crystals are thus of great importance for the outcome of the solar cell performance. The reaction kinetics between $\mathrm{PbI}_{2}$ and MAI is, however, still not perfectly understood. It has been observed that the reaction kinetics of perovskite phase formation is considerably fast, ${ }^{6}$ however, which solvent molecules that actually participate in the reaction and which work as a catalyst is still under discussion.

The dissolution of $\mathrm{PbI}_{2}$ is one of the processes that facilitates the formation reaction of the perovskite phase. ${ }^{7} \mathrm{PbI}_{2}$ is an anisotropic semiconductor that consists of hexagonal layers (I-Pb-I) where the bonding between the layers is weak (van der Waals) and the material has no destructive phase transition between room temperature and the melting point. Small solvent molecules can thereby coordinate with $\mathrm{Pb}$ (II) and partially replace iodine to ligate with lead in an intercalation process during the dissolution. Early on researchers tested many solvent candidates for perovskite formation from $\mathrm{CH}_{3} \mathrm{NH}_{3} \mathrm{I}$ and $\mathrm{PbI}_{2}$. In the first successful tests to form organolead halide perovskite by Kojima et al., ${ }^{8}$ a one-step solution with $\gamma$-butyrolacetone as a solvent

\footnotetext{
*Electrochemical Society Student Member.
}

${ }^{\mathrm{z} E}$ E-mail: Malin.Johansson@kemi.uu.se was used and spin-coated on $\mathrm{TiO}_{2}$ substrate to form perovskite. The currently most common solvents for dissolving $\mathrm{PbI}_{2}$ are dimethylformamide DMF and dimethylsulfoxide DMSO, usually a mixture of both. ${ }^{9}$ In the one-step process the MAI is mixed with the dissolved $\mathrm{PbI}_{2},{ }^{10}$ forming perovskite films upon spincoating and heating. In the two-step process $\mathrm{PbI}_{2}$ thin-films are dipped into MAI dissolved in isopropanol. Methylammonium and iodide ion are inserted to form the perovskite. ${ }^{11}$ The challenge for the two-step process is to fully convert a dense layer of $\mathrm{PbI}_{2}$ to perovskite. ${ }^{12}$ The long reaction time can remove some of the perovskite from the surface. The formation of perovskite is sensitive to exterior influences and it can convert back to the precursors with rise of temperature ${ }^{13}$ and in the presence of moisture. ${ }^{14} \mathrm{MAPbI}_{3}$ Perovskite is also known to have reproducibility problems which needs a careful combination of solvents. ${ }^{15}$ Using a mixture of solvents to control the reaction kinetics for optimized film formation is thereby one of the most engaging topics right now, which has resulted in high power conversion efficiency for the solar cells. ${ }^{15}$

It is also well known that the long term stability remains an issue for the perovskite material. For the development of long lived devices, encapsulation and stable electron and hole transport materials are important. However, to develop stable and sustainable protocols to produce the perovskite materials is also essential. Substantial work on molecular design of the organic species and corresponding structure change from different organic species are expected to further improve the device stability of perovskite solar cells. ${ }^{7}$ The toxicity of the solvents used in the preparation procedure, however, may be a problem in a large scale production, and finding alternative solvents for the production of solar cells is therefore also important. In this study we report the step wise crystal growth of $\mathrm{MAPbI}_{3}$ using the environmentally friendly isopropanol as a solvent in a one-step process, inverse to the most common techniques, by first dissolving MAI and then adding $\mathrm{PbI}_{2}$ powder, leading to an instantaneous reaction to $\mathrm{MAPbI}_{3}$ perovskite. The material is thoroughly characterized, utilizing X-ray and electron diffraction as well as a wide set of different microscopic and spectroscopic techniques. The results in this paper give new insight into the crystal chemistry of the perovskite formation from low dimensional crystals to micro crystals with a new inverse process based on an environmentally friendly route.

\section{Experimental}

Sample preparation.-A $0.67 \mathrm{M}$ perovskite suspension was prepared by dissolving $1 \mathrm{mmol}(159 \mathrm{mg})$ methyl ammonium iodide (MAI) in $1.5 \mathrm{~mL}$ isopropanol and then adding $1 \mathrm{mmol}(462 \mathrm{mg})$ lead iodide $\left(\mathrm{PbI}_{2}\right)$ powder under strong stirring (all chemicals were 


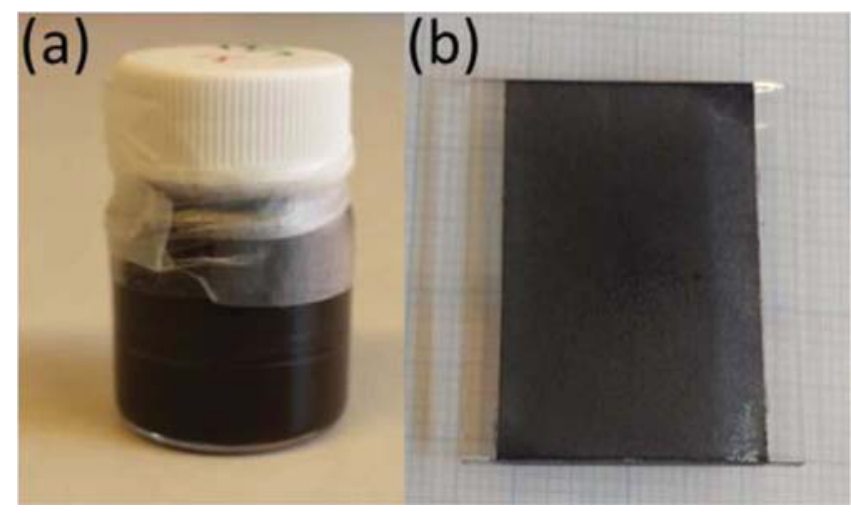

Figure 1. a) $\left(\mathrm{MAPbI}_{3}\right)$ perovskite suspension in isopropanol b) spin-coated $\mathrm{MAPbI}_{3}$ suspension on a glass substrate.

of pro-analysis (PA) and purchased from Sigma-Aldrich). The solution immediately turned from transparent to black while pouring $\mathrm{PbI}_{2}$ into the isopropanol MAI mixture. All reactions were carried out at room temperature. The triiodide perovskite $\mathrm{CH}_{3} \mathrm{NH}_{3} \mathrm{PbI}_{3}$ suspension was synthesized from different molar ratio of the precursors for comparison; One stoichiometric solution with (1:1) molar ratio of $\mathrm{CH}_{3} \mathrm{NH}_{3} \mathrm{I}$ and $\mathrm{PbI}_{2}$ and two non-stoichiometric solutions with excess and lack of methylammonium iodide having molar ratio of (2:1) and (0.5:1), respectively. These perovskite particles were much more stable both in the suspensions and as a spin-coated film than conventional $\mathrm{MAPbI}_{3}$ perovskite thin films and could stand moisture in the air for several weeks. The stability is comparable to colloidal quantum dot perovskites used within optoelectronics $\left(\mathrm{CsPbX}_{3}, \mathrm{X}=\mathrm{Cl}, \mathrm{Br}\right.$ and I). ${ }^{16,17}$

For optical and morphology characterization a thin-film perovskite layer was spin-coated, $2 \mathrm{k}$ rpm for $10 \mathrm{~s}$, on object glasses (Thermo Scientific). The perovskite suspension in a glass jar and a spin-coated glass substrate with a thin film of single crystalline perovskite grains are depicted in Figs. 1a and 1b, respectively.

Material characterization.-The crystal formation was studied using a JEOL (Model JEM-2100F) field emission transmission electron microscope (FE-TEM) with electron diffraction functions, operating at $200 \mathrm{kV}$ and probe size under $0.5 \mathrm{~nm}$. For classification of the Bravais lattice from the electron diffraction using TEM, the Laue condition was used, the reciprocal lattice vector $\boldsymbol{H}$ corresponds to a Bragg reflection from the family of direct lattice planes $[h k l]$ perpendicular to $\boldsymbol{H}$;

$$
\left|H_{h k l}\right|=\frac{1}{d_{h k l}}
$$

where $d_{h k l}$ is the distance between the lattice planes.

The crystalline structure and phase of the $\mathrm{MAPbI}_{3}$ thin films were determined by grazing incidence X-ray diffraction (GIXRD), using a Siemens D5000 $\theta-2 \theta$ instrument, employing parallel-plate sollers with a resolution of $0.3^{\circ}(2 \theta)$. X-ray diffraction (XRD) patterns were recorded from the crystalline perovskite grains of the evaporated isopropanol solution (powder) using a Bruker D8 TwinTwin instrument with Bragg Brentano set-up and resolution of $0.05^{\circ}(2 \theta)$. The $\mathrm{Cu} K_{\alpha 2}$ influence was corrected for using the program software Eva.

The diffraction angle $\theta$ was determined using Bragg's law; $n \lambda=$ $\mathrm{d}_{\mathrm{hkl}} \sin \theta$, where $\lambda=1.54056 \AA$ is the $\mathrm{CuK} \alpha_{1} \mathrm{X}$-ray wavelength.

The lattice constants $\boldsymbol{a}$ and $\boldsymbol{c}$ were calculated using the unit cell for the tetragonal phase using;

$$
d_{h k l}=\frac{1}{\sqrt{\frac{h^{2}+k^{2}}{a^{2}}+\frac{l^{2}}{c^{2}}}}
$$

For the small amount of cubic phase occurring in the structure the lattice constant a was determined by;

$$
d_{h k l}=\frac{a}{\sqrt{h^{2}+k^{2}+l^{2}}}
$$

The surface morphology and grain sizes of the formed nanostructures were characterized with scanning electron microscopy (SEM), using a LEO 1550 FEG instrument (LEO Electron Microscopy Ltd., Cambridge, UK) with in-lens detector operating at $10 \mathrm{kV}$.

Optical characterization and material properties.-The reflectance and transmittance of a spin-coated layer of perovskite solution on a lab-glass were measured with a Perkin-Elmer Lambda 900 double-beam UV/Vis/NIR spectrophotometer equipped with an integrating sphere and a Spectralon reflectance standard. The absorption coefficient $\alpha$ as a function of wavelength $\lambda$ was determined by spectrophotometry from the special absorption ${ }^{18}$

$$
\alpha(\lambda)=\frac{1}{d} \ln \left(\frac{1-R(\lambda)}{T(\lambda)}\right)
$$

where $d$ is the thickness of the film. The film thickness, $d$, was determined from surface profilometry, Dektak XT (Bruker) and it was found to be $d_{(1: 1)}=690 \mathrm{~nm}$ for the stoichiometric sample and $d_{(2: 1)}=$ $700 \mathrm{~nm}$ and $d_{(0.5: 1)}=500 \mathrm{~nm}$ for the nonstoichiometric samples.

Photoluminescence emission measurements were performed using a Horiba Jobin Yvon Fluorolog instrument (Model FL3-222). The signal was automatically corrected for wavelength dependent instrument sensitivity and normalized with a reference signal to compensate for lamp fluctuations. The measurements were performed in backscattering geometry employing $30^{\circ}$ angle of incidence of the excitation beam with respect to the surface normal and the detection optics aligned along the surface normal. A monochromator slit width resulting in a spectral resolution of $5 \mathrm{~nm}$ was used.

Further material properties were determined using Raman spectra which were recorded with a Renishaw InVia Raman spectrometer with $1 \mathrm{~cm}^{-1}$ resolution using a frequency-doubled YAG laser (532 $\mathrm{nm}$ ) and 1800 lines/mm grating. A 50x objective was used and gave a laser spot with $3 \mu \mathrm{m}$ diameter with $50 \mu \mathrm{W}$ intensity on the sample. A notch filter was used, blocking the plasma line $10 \mathrm{~cm}^{-1}$ into the Stokes part of the spectra.

\section{Results and Discussion}

Crystal formation.-Within seconds, the solution turns black when adding $\mathrm{PbI}_{2}$ powder into the dissolved MAI in isopropanol (Fig. 1a). Isopropanol acts as good solvent for the organic part (MAI) due to solvent-solute hydrogen bond interactions, which result in $\mathrm{MA}^{+}$ and $\mathrm{I}^{-}$ions. $\mathrm{PbI}_{2}$, on the other hand is nearly insoluble in isopropanol. The $\mathrm{PbI}_{2}$ crystal structure has a layered structure allowing intercalation of MA additionally assisted by the sheet like morphology, see Fig. 3b, leading to high accessible surface for reactions. The ions react very fast when they are inserted into the isopropanol solution of MAI. The morphology and size of the particles change significantly, see SEM pictures, demonstrating that the reaction is not only the insertion of $\mathrm{MA}^{+}$and $\mathrm{I}^{-}$ions into $\mathrm{PbI}_{2}{ }^{11}$ but is also mediated by the benign environment of the solvent molecules.

The low solubility of $\mathrm{PbI}_{2}$ in alcohols will minimize asymmetric interactions with ions from the solvent itself during their assembly into crystal form. In this approach, the dissolved $\mathrm{MA}^{+}$and $\mathrm{I}^{-}$will then react with the undissolved $\mathrm{PbI}_{2}$ primarily via intercalation assisted by the isopropanol and form the $\mathrm{MAPbI}_{3}$ perovskite with a rather low activation energy due to the high solubility of MAI. ${ }^{19}$

In Figure 2 below it is possible to follow the crystal growth from low dimensional nucleating quantum dots (QDs) to large grains from the same sample preparation (1:1) by following the (a), (b), (c), (d), (e) and (f) images. In Figs. $2 a$ and $2 b$ the solution has just been prepared and the QDs have been separated with extra solvent to clearly see the particle formation in the TEM microscope. The perovskite QD, seen in Fig 2a, reveal an icosahedron form viewed from above with five 

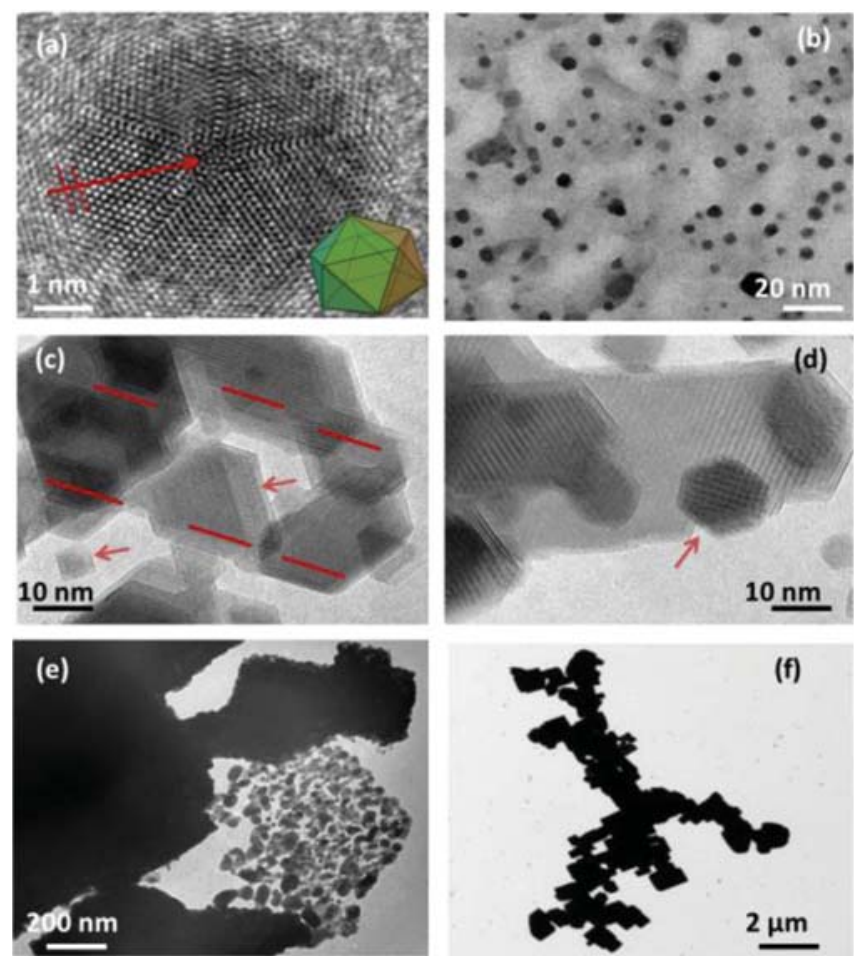

Figure 2. Organized crystal formation of the perovskite (1:1) solution, from small quantum dots to larger grains (a) A $6 \mathrm{~nm}$ sized QD formed like an icosahedron seen from above (b) An overview of the QD particles (c) and (d) The particles are highly crystalline and formed into different shapes (e) smaller particles agglomerate into larger grains (f) Square grains of the structured perovskite.

equilateral triangular faces meeting at each vertex in total 20 facets. Layers of atoms are stacked into a dense packed pyramid like shape. The atoms are organized into a version of a close packed system, an arrangement often occurring in an octahedral geometry. Seen from above, the third layer of atoms is in the same row as the first layer, and the fourth layer in the same row as the second etc. This can be seen on the facets, maybe most clear on the one to the left. The white dots are the atoms and if one follows a radial line to the center of the icosahedron, enhanced with a red arrow, it is possible to see that at every second row of atoms are inline to the center. To guide the eye to see the rows with the inline atoms, two red lines have been drawn. In the z-direction, pointing out of the plane of the paper, the atoms are shifted to adjust into the close packed form. Figure $2 \mathrm{~b}$ shows an overview of a QD area where most of the particles are below $10 \mathrm{~nm}$ semblance to form hexagonal or pentagonal shapes. Figs. 2c and $2 \mathrm{~d}$ show less separated QD and the particles with their atoms seem to organize in a remarkable way. There are cubic, quadratic, hexagonal, triangular and pentagonal shapes, where the hexagonal is on average most occurring, a few of the shapes pointed out with arrows. The atoms are well structured in each particle and the grains appear to be connected since the direction of atoms is equal in between the grains and turns up in the same straight lines, emphasized with red lines in Fig. 2c. At this point the material is distinctly crystalline. Fig 2e shows how the particles agglomerate into larger clusters, and end up in an energetic beneficial cubic formation as the crystal grows, Fig. 2f, which is also seen in the SEM images below (Fig. 3a).

Structure and morphology.-To investigate the microstructure and morphology of spin-coated perovskite thin films, prepared from the isopropanol dispersion, scanning electron microscopy (SEM) was used. The images showed in Fig. 3 were performed at different magnifications, an overview to the left and a zoom in on the grains to the right of each sample.
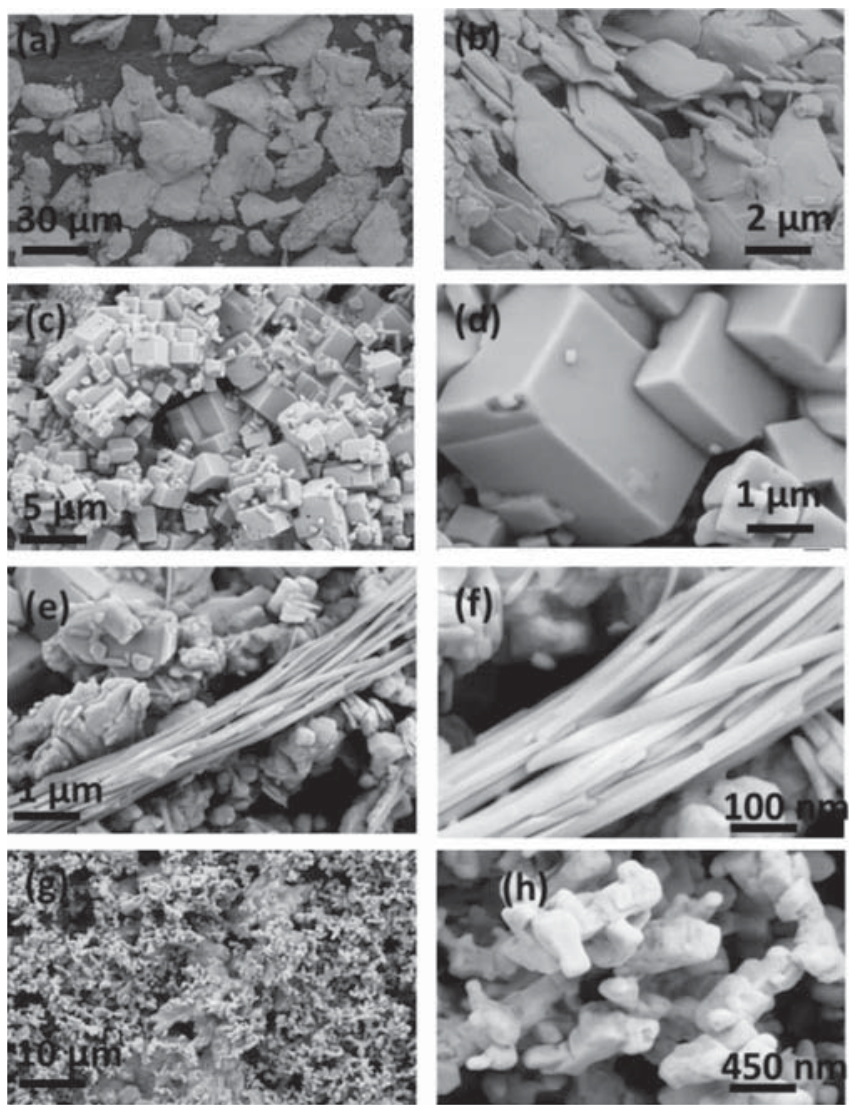

Figure 3. SEM images of the $\mathrm{PbI}_{2}$ powder and the samples with different molar ratio, observe the different magnifications, lower to the left and higher to the right; (a) and (b) $\mathrm{PbI}_{2}$, (c) and (d) stoichiometric (1:1) $\mathrm{MAPbI}_{3}$ showing square cubic shaped grains, (e) and (f) non-stoichiometric $(0.5: 1)$ sample showing rods and square grains, $(\mathrm{g})$ and $(\mathrm{h})$ grains from the (2:1) sample showing connected grains in a net like morphology.

Figures $3 \mathrm{a}$ and $3 \mathrm{~b}$ show the $\mathrm{PbI}_{2}$ powder and when closing in on the $\mathrm{PbI}_{2}$, it is seen that it contains stacks with thin layers of $\mathrm{PbI}_{2}$ sheets close packed together, Fig. 3b. Two adjacent sheets are bound together with weak van der Waals forces in the repeated I-Pb-I sandwich formation. When the $\mathrm{PbI}_{2}$ powder is inserted in the isopropanol solution containing $\mathrm{MA}^{+}$and $\mathrm{I}^{-}$a total transformation occurs. The result is observed in Figs. $3 \mathrm{c}$ and $3 \mathrm{~d}$ which shows the stoichiometric (1:1) $\mathrm{MAPbI}_{3}$ perovskite. Different sizes of well-shaped cubic grains with very clear lines and edges are formed. The distribution of different grain sizes is fairly large. In the zoom in image, Fig. 3d, an approximately $4 \mu \mathrm{m}$ grain is depicted. Figs. $3 \mathrm{e}$ and $3 \mathrm{f}$ show the nonstoichiometric sample $(0.5: 1)$ with a variation of shapes. It contains approximately $30 \%$ rods between the cubic grains. $\mathrm{PbI}_{2}$ has been reported to form rods ${ }^{20,21}$ and it is reasonable to believe that the rods only contain $\mathrm{PbI}_{2}$ also since the concentration of $\mathrm{PbI}_{2}$ is twice as high as MAI and no rods are seen in the stoichiometric sample. It has been observed in previous reports that $\mathrm{PbI}_{2}$ transform into rod-like particles in alcohols..$^{20}$ The rods can be up to $10 \mu \mathrm{m}$ long and $40 \mathrm{~nm}$ wide. The cubic grains are moreover surrounded by small rods in the $100 \mathrm{~nm}$ range. Figures $3 \mathrm{~g}$ and $3 \mathrm{~h}$ show the (2:1) sample with cubic grains having soft and a bit round edges. The grain size is in the 400-600 $\mathrm{nm}$ range and they seem to be more connected in the structure.

The phase purity of the grown crystals in solution was confirmed by X-ray diffraction (XRD). XRD was performed on the spin-coated thin films using a grazing incident setup, and on powder ground from batches of crystals with a Bragg-Brentano setup, both, working at $2 \theta$ performance.

The main contribution of crystallinity from all the samples can be derived to the body centered tetragonal lattice with space group 

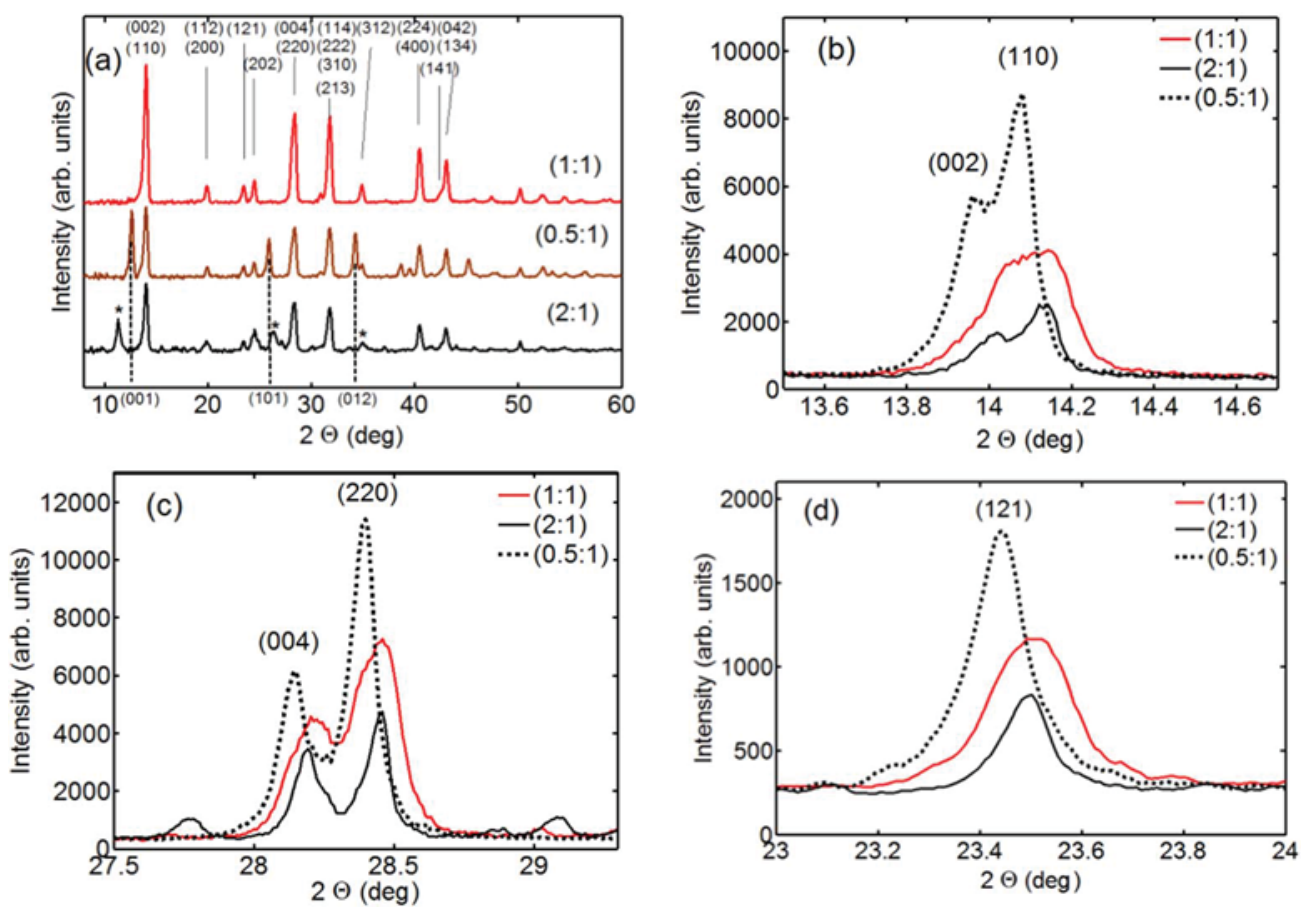

Figure 4. X-ray diffraction pattern of perovskite $\mathrm{MAPbI}_{3}$ structure for stoichiometric and non-stoichiometric samples at molar ratio (1:1), (2:1) and (0.5:1), following the reference ${ }^{24}$ (a) an overview; the tetragonal lattice planes for $\mathrm{MAPbI}_{3}$ denoted above, seen in all four samples, and $\mathrm{PbI}_{2}$ hexagonal phase, seen in the $(0.5: 1)$ sample below the graph. The star indicates peaks from the contribution of crystallized methylammonium iodide. Figure (b), (c) and (d) shows zoom in on powder diffraction from a few characteristic peaks from the $\mathrm{MAPbI}_{3}$ structure.

$(\mathrm{I} / \mathrm{mcm})$ with some fraction from the cubic lattice $(\mathrm{Pm} 3 \mathrm{~m})$. The typical perovskite diffraction peaks have a surprisingly strong intensity also for the non-stoichiometric samples. Fig. 4a is an overview of the samples measured with a grazing incidence XRD for thin films. Here it is impossible to separate the narrow peaks at for instance $2 \theta=$ $14^{\circ}$ coming from (110) and (002) diffraction planes in a tetragonal phase.

However, Figs. 4b, 4c and 4d are showing powder diffraction, with a resolution of FWHM $0.05^{\circ}$, from the single crystals of the three samples with focus on the peaks that are typical for the $\mathrm{MAPbI}_{3}$ perovskite. In these measurements the peaks from the diffraction planes (004) and (220) at $2 \theta=28.15^{\circ}$ and $28.4^{\circ}$, seen in Fig. 4c, are clearly separated and can indeed prove the tetragonal phase, in particular for the $(0.5: 1)$ sample. The diffraction peaks are in good agreement with the calculated diffraction described by T. Baikie et al. ${ }^{22}$ and other detailed studies of the diffraction of the perovskites. ${ }^{23}$

The cell parameters for the $(0.5: 1)$ samples were found to be $\mathrm{a}=$ $\mathrm{b}=8.89 \AA$ and $\mathrm{c}=12.69 \AA$. A peak shift is detected in the (002) and (004) plane diffraction of the (1:1) and (2:1) samples compared to the $(0.5: 1)$ sample, seen in the electron diffraction in Figs. $5 b$ and $5 \mathrm{c}$ respectively. The cubic peak position of the (002) diffraction plane is reported to be at $2 \theta=28.26^{\circ}{ }^{23}$ This is close to the diffraction angle of sample (1:1) and (2:1) at $28.24^{\circ}$ and $28.21^{\circ}$, see Table I below. Fig. $4 d$ shows the diffraction peak (121) which only belongs to the tetragonal phase. Since the powder samples come from a solution which just has been evaporated, we can assume random orientation of the crystals. From the relative intensities of this peak we can approximate the ratio of the tetragonal phase compared with the cubic phase. If we assume that sample $(0.5: 1)$ has a pure tetragonal phase (because of its highest intensity and suggested from Raman data discussed later), for sample $(1: 1) 65 \%$ is calculated to be tetragonal phase and for sample (2:1) $46 \%$, with the rest being cubic. At room temperature $\mathrm{MAPbI}_{3}$ is known to be tetragonal and changing to the cubic phase at approximately $54^{\circ} \mathrm{C} .^{23}$ In this report we find a contribution from cubic phase at room temperature. It seems like the cubic phase is more stable at the surface and has been reported by Protesescu et al. where all the $\mathrm{CsPbX}_{3}$ QD particles forms cubic phase instead of tetragonal or orthorhombic for the perovskite lattice. ${ }^{17}$

Table I shows the calculated $d_{(\mathrm{hkl})-\text { spacing }}$ and lattice constants, using formula 3 and 4 , originating from the experimental $\mathrm{X}$-ray powder diffraction. In particular sample $(0.5: 1)$ agrees very well with the tetragonal phase. ${ }^{25}$ The peak positions from Fig. $4 \mathrm{~b}$ in the range $13.8-14.3^{\circ}$ and Fig. $4 \mathrm{c}$ in the range $27.5-29.5^{\circ}$ have been used. The peak from the (002) plane in the (1:1) sample overlaps with the (110) diffraction and is seen more as a shoulder. An approximate peak position for the (002) has been used in this sample. The peak shift of
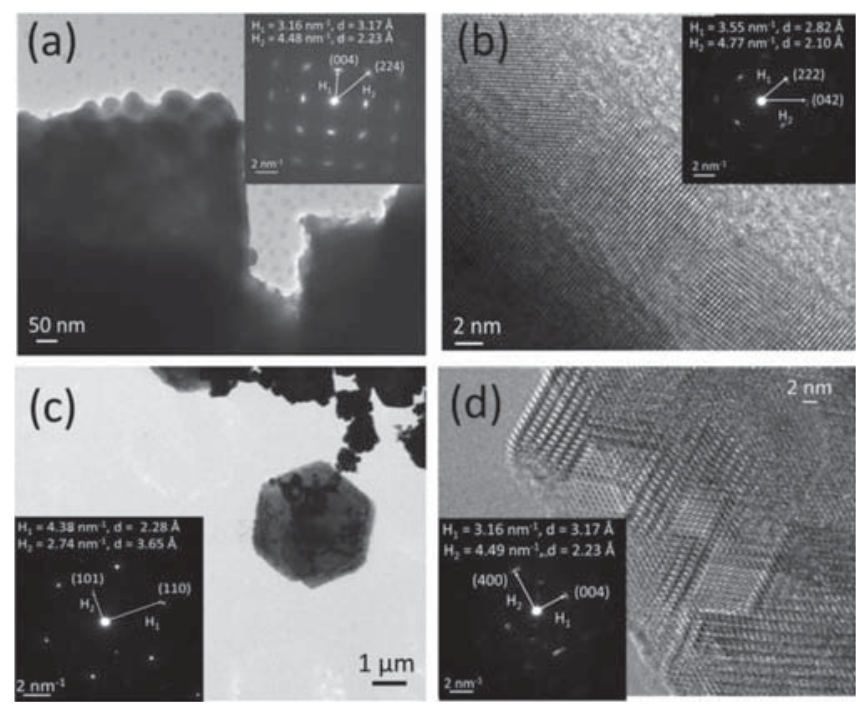

Figure 5. TEM images and ED; (a) and (b) electron diffraction from the area on the image from the stoichiometric (1:1) sample, (c) ED from the hexagonal character in the $(0.5: 1)$ sample and (d) an ED and image from the $(2: 1)$ sample. 


\begin{tabular}{|c|c|c|c|c|c|c|c|c|c|}
\hline Sample (MAI:PbI ${ }_{2}$ ) & $(h k l)_{\text {tet }}$ & $(h k l)_{\mathrm{cub}}$ & $2 \theta_{\text {tet }}$ & $2 \theta_{\text {cub }}$ & $d_{t e t}(\AA)$ & $d_{c u b}(\AA)$ & ratio $_{\text {tet }}$ & $\mathbf{c}_{t e t} \mathbf{a}_{c u b}(\AA)$ & $\mathbf{a}_{t e t}$ \\
\hline \multirow[t]{4}{*}{$(1: 1)$} & $(002)$ & $(100)$ & 13.97 & 14.05 & 6.34 & 6.30 & 0.65 & 12.68 & 6.30 \\
\hline & (110) & & 14.15 & & 6.26 & & & 8.85 & \\
\hline & (004) & $(200)$ & 28.15 & 28.24 & 3.17 & 3.16 & & & \\
\hline & $(220)$ & & 28.46 & & 3.14 & & & & \\
\hline \multirow[t]{4}{*}{$(2: 1)$} & $(002)$ & $(100)$ & 13.98 & 14.02 & 6.33 & 6.32 & 0.46 & 12.67 & 6.32 \\
\hline & (110) & & 14.15 & & 6.26 & & & 8.86 & \\
\hline & $(004)$ & $(200)$ & 28.15 & 28.21 & 3.17 & 3.17 & & & \\
\hline & $(220)$ & & 28.46 & & 3.14 & & & & \\
\hline \multirow[t]{4}{*}{$(0.5: 1)$} & $(002)$ & & 13.96 & & 6.34 & & 1 & 12.69 & \\
\hline & (110) & & 14.08 & & 6.29 & & & 8.89 & \\
\hline & $(004)$ & & 28.14 & & 3.17 & & & & \\
\hline & $(220)$ & & 28.40 & & 3.14 & & & & \\
\hline
\end{tabular}

the (002) plane determined both the tetragonal $\mathbf{c}$ lattice and exploited as a cubic (100) diffraction giving the a lattice distance. Both of them are probable and give realistic values; reflecting a shift thought of as change of the lattice distance in the c-direction when the tetragonal structure approaches a more cubic character. The other suggestion is that sample (1:1) and (2:1) contains an undefined ratio of cubic phase, in that case the cell parameters for the $\mathrm{a}=\mathrm{b}=\mathrm{c}=6.30 \AA$ and $6.32 \AA$ for the (1:1) and (2:1) sample, respectively. In all, there is a shorter bond length between the atoms in the c-direction for the stoichiometric (1:1) and non-stoichiometric (2:1) than the nonstoichiometric (0.5:1) sample.

Fig. 5a shows a part of a grain and the inset shows electron diffraction (ED) from that part. The ED has a square shaped pattern originating from the tetragonal perovskite phase confirming the whole grain is essentially a single-crystal. The wavelength of the electrons is much shorter than the x-rays. Therefore, the radius of the Ewald sphere is much larger and more reflections are observed by ED than by XRD. The length of the scattering vectors to each diffraction maximum reveals the plane separation for the corresponding Miller index (hkl).

With Bragg's law, the diffraction angle from the X-ray diffraction can be compared with the $d_{(\mathrm{hkl})}$ value. In Fig. 5a, the vector denoted $\mathrm{H} 1$ gives $d_{(004)}=3.17 \AA$ using formula [2] corresponding to the angle of $2 \theta=28.12^{\circ}$. From the d-spacing we can derive the lattice constant c from formula [2] for the tetragonal phase which gives a value of 12.68 $\AA$. This is a reasonable value for the unit cell in the c-direction of the tetragonal phase. For comparison, the d- spacing can be used with the cubic formula 3 , giving an a-axis of $12.68 \AA$ which is too large for the cubic unit cell. Here it can be stated that this is a tetragonal phase. At $45^{\circ}$ angle the $\mathrm{H}_{2}$ vector corresponds to $d_{(224)}=2.23 \AA$, which corresponds to $2 \theta=40.34^{\circ}$. These values can be compared with the $\mathrm{X}$-ray diffraction peaks typical for the $\mathrm{MAPbI}_{3}$ perovskite structure in Fig. 4c.

Fig. $5 b$ shows a TEM image of the edge of a cube. It is seen that the atoms are lined up in rows. The ED in the inset of the image shows a single crystalline pattern taken from the edge area in the middle of Fig. 5b. The distance between the planes corresponding to $\mathrm{H} 1$ is $d_{(222)}=2.82 \AA$ which is comparable with the $2 \theta=31.72^{\circ}$ and $\mathrm{H} 2$ gives $d_{(042)}=2.10 \AA$, corresponding to $2 \theta=43.14^{\circ}$. The electron diffraction convincingly matches the X-ray diffraction pattern. Small shifts in angle and d spacing can be due to errors in both XRD and ED measurements, (the sample has to be orthogonal to the incident $\mathrm{X}$-ray or electron beam to calculate the exact distance between the d-planes). An important observation connected to the accuracy of the measured reciprocal space vector dependent on the spot-size in ED: small changes in length of the vector $\mathrm{H}$ shifts the diffraction $2 \theta$ angle fairly much. However, the accuracy of these measurements and the agreement between ED and XRD is rather high.

Figure $5 \mathrm{c}$ shows a thin $2 \mathrm{D}$ hexagonal particle occurring, to a low amount, in the $(0.5: 1)$ sample. This is probably a $\mathrm{PbI}_{2}$ particle with the $\mathrm{Pb}$-ion surrounded by iodine. ${ }^{26}$ The ED from only that particle is seen in the inset of Fig. 5c. The $d_{(101)}=2.28 \AA$ and the $d_{(110)}$ $=3.65 \AA$ correspond to an angle of $2 \theta=39.49^{\circ}$ and $2 \theta=24.37^{\circ}$ respectively. The $2 \theta=24.37^{\circ}$ does not agree completely with the (110) plane for the $\mathrm{PbI}_{2}$, which should be placed at approximately $2 \theta$ $=26^{\circ}$ according to the experimental XRD data. The reason for this is still under discussion.

Fig. $5 d$ shows the $(2: 1)$ sample at the corner of a grain. The atoms are organized in an interesting crystalline form. The ED in the inset shows a single crystalline pattern quite similar to Fig. 5a except for the $90^{\circ}$ angle between the diffraction spots. This might be a type of $2 \mathrm{D}$ network where the organic ions become interdigitating bilayers of intercalated cations. ${ }^{27}$ The spots correlate to $d_{(004)}=3.17 \AA$ giving $2 \theta=28.12^{\circ}$ and $d_{(040)}=2.23 \AA$ giving $2 \theta=40.41^{\circ}$, in good agreement with the XRD data.

Perovskite optoelectronic properties.-UV/vis/NIR spectrophotometry and photoluminescence.-Spectrophotometry was used to measure the transmittance and reflectance of the spin-coated perovskite solutions on glass in the range $330-1400 \mathrm{~nm}$. The emission spectra were measured with a photoluminescence spectrometer in the range $650-900 \mathrm{~nm}$ using reflecting mode setup.

Fig. 6a shows the total transmittance of the three samples. The samples have a transmittance of $10-25 \%$ at wavelengths below 800 $\mathrm{nm}$, and $35-50 \%$ at wavelengths above $800 \mathrm{~nm}$. The reflectance, Fig. $6 \mathrm{~b}$, is low at short wavelengths and increases after $800 \mathrm{~nm}$ up to $60 \%$. The high reflectance is caused by a large diffuse reflective part due to light scattering. This can be due to the rather large single crystalline grains which are disorganized on the surface after spin coating. The specular reflective part is close to zero over the whole range.

The absorption coefficient $\alpha$, seen in Fig. $6 c$, has been calculated using formula [1]. It shows a sharp change of absorption at $800 \mathrm{~nm}$ where the bandgap excitation of electrons into the conduction band occurs. High absorption is observed in the range $330-800 \mathrm{~nm}$. By extrapolating the linear region of the absorption edge to the $\mathrm{x}$-axis intercept, the optical bandgap was estimated to $E_{\mathrm{g}}=1.55 \mathrm{eV}$. The very sharp step of absorption is similar to the single crystal absorption behavior described by D. Shi et al., ${ }^{28}$ where the perovskite crystals have been synthesized using an anti-solvent vapor assisted method. The shape of the curve differs from absorption spectra of non-single crystals of perovskite. We believe that this is not due to saturation since there is a small amount of light that is still transmitted in the 330-800 $\mathrm{nm}$ range. The shape is more likely to come from the morphology of the surface and pin-hole effects.

Even if there is twice as much of $\mathrm{PbI}_{2}\left(\mathrm{E}_{\mathrm{g}}=2.3-2.55 \mathrm{eV}\right)$ in the (0.5:1) sample, a very small absorption caused by the $\mathrm{PbI}_{2}$ contribution can be observed. Absorption due to $\mathrm{Pb}^{2+}$ complexes such as triiodoplumbate $\left(\mathrm{PbI}_{3}{ }^{-}\right)$and tetraiodoplumbate $\left(\mathrm{PbI}_{4}{ }^{2-}\right)$, in the 350 $450 \mathrm{~nm}$ region $^{29}$ is not observed, however, there is a shoulder in the (0.5:1) sample at $500 \mathrm{~nm}$ which indicates on an excess of $\mathrm{PbI}_{2}{ }^{30}$ Usually, there is a small exponential slope in semiconductors after 

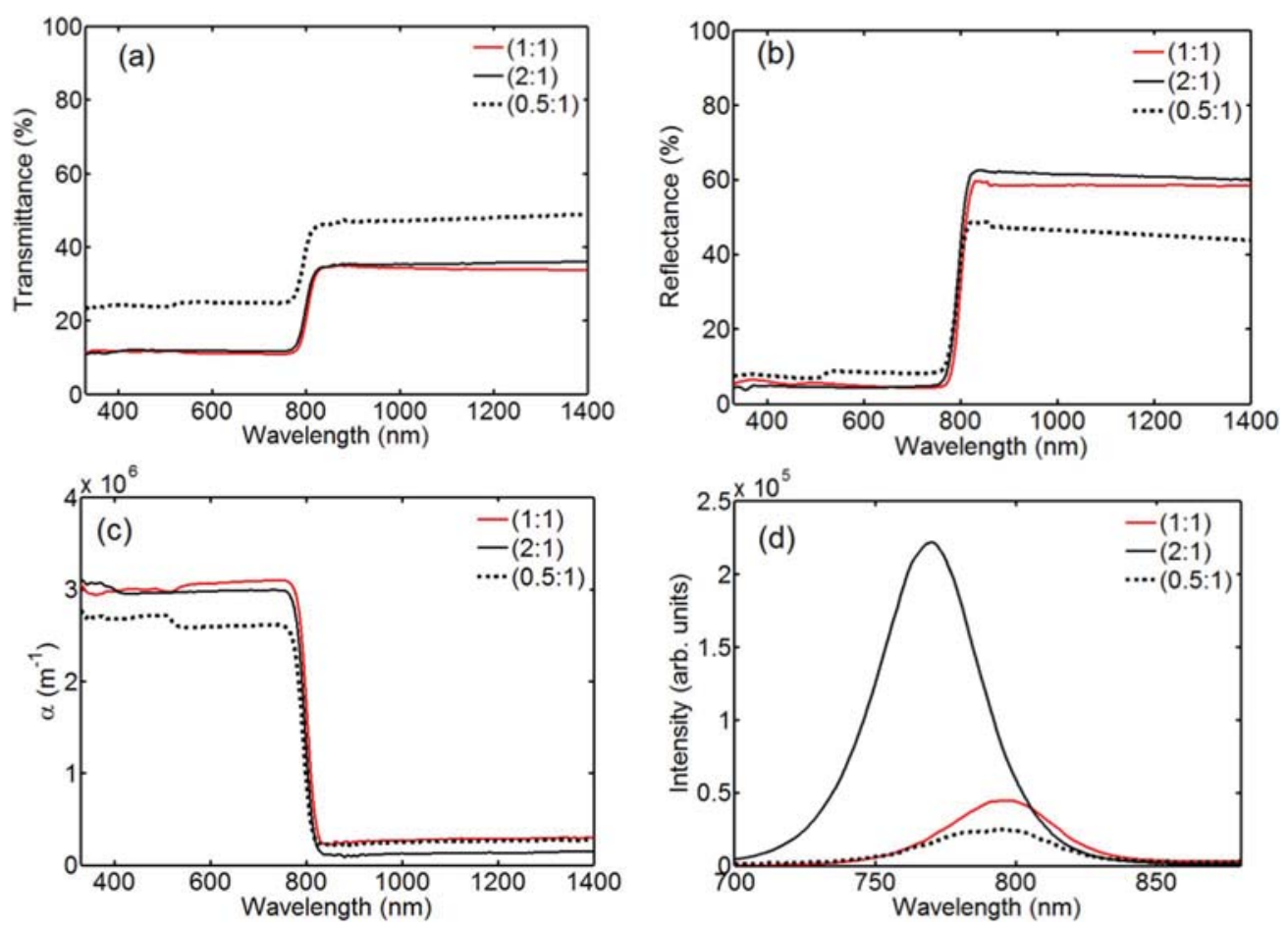

Figure 6. UV/vis/NIR spectrophotometry and photoluminescence of the four samples at different molar ratio with spin coated solution on a lab glass (a) transmittance, (b) reflectance, (c) absorption coefficient $\alpha$ and (d) emission spectra at excitation wavelength $\lambda_{\mathrm{ex}}=600 \mathrm{~nm}$.

the absorption edge; for instance in oxides like $\mathrm{WO}_{3}{ }^{31}$ and $\mathrm{TiO}_{2}$ as well as $\mathrm{Si}$ and $\mathrm{GaAs},{ }^{32}$ the so called absorption tail states (or Urbach tail). ${ }^{33}$ This exponential behavior is attributed to structural disorder in the material. The absorption from the perovskite thin films, seen in Fig. $6 \mathrm{c}$ in the range $800-1400 \mathrm{~nm}$, has no exponential tendency where optically detectable deep states are present, which suggests a low degree of structural disorder in the $\mathrm{MAPbI}_{3}$ perovskite structure and, therefore, high crystallinity.

Fig. 6d shows an emission spectrum with an excitation wavelength of $\lambda_{\text {ex }}=600 \mathrm{~nm}$. The intensity from the emission varies between the samples with highest intensity from the $(2: 1)$ sample where the concentration of the MAI component is twice as much. It is also observed that the peak is blue shifted. The peak is expected to be positioned at approximately $800 \mathrm{~nm}$ where the electronic band transition occurs or at longer wavelengths due to excitonic binding energies. However, now the peak position is at $760 \mathrm{~nm}$. One probable explanation of this behavior is the larger amount of MAI, which can be compared with the explanation of Papavassiliou et al. ${ }^{34}$ The article describes how to tune the PL band in the range 400 to $700 \mathrm{~nm}$ with the ratio of mass containing of the organic part in between the metal compounds. The perovskite formation of sample (2:1) fits very well to the 2D structure mentioned before. The peak position has a comparable placement with the emission peak of $\left(\mathrm{CH}_{3}\left(\mathrm{CH}_{2}\right)_{3} \mathrm{NH}_{3}\right)_{2}\left(\mathrm{CH}_{3} \mathrm{NH}_{3}\right)_{3} \mathrm{~Pb}_{4} \mathrm{I}_{14} \cdot{ }^{27}$

The comparably low PL-band intensity from the (1:1) and (0.5:1) samples might be due to the high crystallinity and low amount of distortion, since PL partly originates from defects in the material. ${ }^{27}$ This might also be one of the reasons for the high PL intensity of the (2:1) sample where there occur other structural components, seen in Fig. 6a. The defects are likely to play a crucial role in the PL spectra for the non-stoichiometric samples.

The variation of the $\mathrm{PbI}_{2} / \mathrm{CH}_{3} \mathrm{NH}_{3} \mathrm{I}$ composition ratio in the solution has been reported to have the best electronic properties at the molar ratio of $0.6-0.7 .^{35}$ This can be compared with the (2:1) sample, which might have a higher doping concentration that also can be an advantage for the charge transport in the solar cell.
Raman spectroscopy.-The vibration modes of the single crystalline thin films have been investigated by Raman scattering. Fig. 7 shows the result from the three samples (1:1), (2:1) and (0.5:1). At most six bands have been observed in the $50-350 \mathrm{~cm}^{-1}$ region. They are at $70,96,110,140,166$ and $214 \mathrm{~cm}^{-1}$. The first normal mode at $70 \mathrm{~cm}^{-1}$ is predicted to be the $\mathrm{Pb}-\mathrm{I}$ asymmetric stretching and gives information of the inorganic component of the material. ${ }^{36}$ The shoulder at $140 \mathrm{~cm}^{-1}$ and the small peak at $166 \mathrm{~cm}^{-1}$ have been related to the MA cation in previous reports. ${ }^{37}$ In an octahedron perovskite structure, one expects the phonon spectrum to contain three transverse optical (TO) and three longitudinal optical (LO) phonon modes. The line at $214 \mathrm{~cm}^{-1}$ is considered to be the second order band corresponding to modes from longitudinal optical (LO) phonons. ${ }^{20}$ Fig. 7a shows the variation of intensity from the samples. It was approximately the same ratio in between the samples also after measuring at different spots on the surface. The lower intensity from the (1:1) and (2:1) is likely due to the fraction of cubic phase and possibly also from the decrease of the c-axis length which causes a smaller dipole moment. The symmetry group which the cubic phase belongs to have no quadratic symmetry element and there will be no vibrations that are Raman active. This fact correlates very well with the ratio of tetragonal phase derived from the relative intensity of the (121) XRD peak in Fig. 4d. Fig. $7 \mathrm{~b}$ shows the normalized (to $96 \mathrm{~cm}^{-1}$ ) Raman intensity to compare the relative intensity of the vibration peaks in the sample. In both figures it is observed that the height between the peak at $96 \mathrm{~cm}^{-1}$ and $110 \mathrm{~cm}^{-1}$ differs. The ratio $\delta$ of sample $(0.5: 1)$ from peak position $96 / 110 \mathrm{~cm}^{-1}$ is $\delta=1.27$, sample $(1: 1) \delta=1.16$ and sample $(2: 1) \delta=1.06$. The higher ratio in between the two peaks of the $(0.5: 1)$ can be attributed to the higher $\mathrm{PbI}_{2}$ concentration which has the most intense peak at $96 \mathrm{~cm}^{-1}$ coinciding with the peak from the perovskite at the same position. However, the higher intensity of the Raman lines situated at low frequencies compared to the higher ones indicates that the crystal is more polarizable at low frequencies. This correlates with the XRD data seen in Fig. 6 where we found that the $(0.5: 1)$ sample has the most typical tetragonal phase with a c-axis of $12.7 \AA$ and a-axis of $8.9 \AA$. This is true only if the transverse optical 

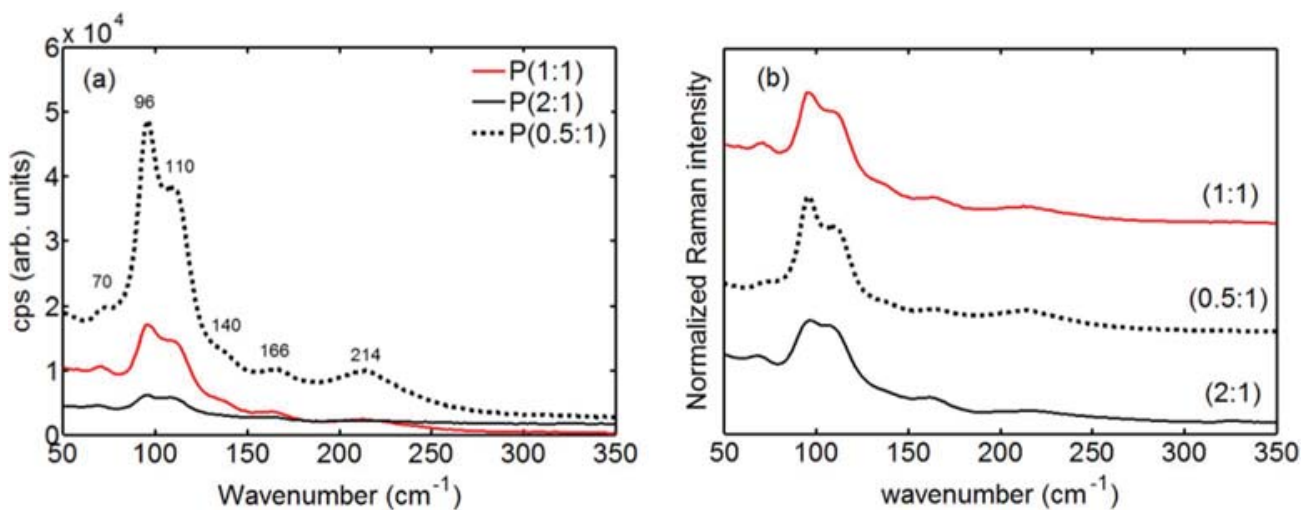

Figure 7. Raman spectra in the $50-350 \mathrm{~cm}^{-1}$ wavenumber range with $\lambda_{\mathrm{exc}}=523 \mathrm{~nm}$ (a) Peak positions and variation in intensity (b) Normalized intensity of the Raman spectra.

mode is situated at $96 \mathrm{~cm}^{-1}$ and the longitudinal optical mode is at $110 \mathrm{~cm}^{-1}$.

\section{Conclusions}

Single crystal formation from quantum dots to micrometer crystals has been followed for a synthesis protocol using a more environmentally friendly route with respect to the solvent. TEM analysis shows small perovskite quantum dots to form and then organize into larger complexes and end up into well-organized single crystals. Comparison of three different molar ratios of $\mathrm{MAPbI}_{3}$ perovskite solution (1:1), $(2: 1)$ and $(0.5: 1)$ was performed at room temperature. The samples were further characterized with electron and X-ray diffraction. The results showed a fraction of cubic phase in the (1:1) sample (35\%) and in the (2:1) sample (54\%) and correlated well with Raman spectroscopy. The optical properties of the single crystalline perovskite particles were analyzed with spectrophotometry and photoluminescence. Both mechanism and progress of crystal formation as well as the final crystallinity are of high importance for the charge transport in the solar cell where the data and analysis presented in this study give knowledge about this in a new and more environmentally friendly route for synthesis of perovskite crystalline films.

The environmental aspect of isopropanol as a solvent for life cycle analysis and waste is significant in solar cell production. We have shown that it is possible to produce quality $\mathrm{MAPbI}_{3}$ perovskite crystals in environmental friendly solvent like isopropanol.

\section{Acknowledgments}

We thank the Swedish Energy Agency, the STandUP for Energy program, the Swedish Research Council (VR), the Swedish Research Council, FORMAS, the Göran Gustafsson Foundation, ÅForsk, and the Knut and Alice Wallenberg Foundation. We also thank Wubeshet Sahle for fruitful discussions during TEM analysis.

\section{References}

1. http://www.nrel.gov/ncpv/images/efficiency_chart.jpg, in (2015).

2. D. B. Mitzi, J. Chem. Soc., Dalton Trans., 1 (2001).

3. D. B. Mitzi, C. D. Dimitrakopoulos, and L. L. Kosbar, Chem. Mater, 13, 3728 (2001).

4. J. D. Currey, The mechanical adaptions of bones, Princeton University Press, New Jersey, UK (1984).

5. G. C. Pavassiliou, Prog. Solid St. Chem., 25, 125 (1997).

6. D. Liu and T. L. Kelly, Nature photonics, 8, 133 (2014).

7. T.-B. Song, Q. Chen, H. Zhou, C. Jiang, H.-H. Wang, Y. M. Yang, Y. Liu, J. You, and Y. Yang, J. Mater. Chem. A, 3, 9032 (2015).
8. A. Kojima, K. Teshima, Y. Shirai, and T. Miyasaka, J. Am. Chem. Soc., 131, 6050 (2009).

9. J. P. Correa Baena, L. Steier, W. Tress, M. Saliba, S. Neutzner, T. Matsui, F. Giordano, T. J. Jacobsson, A. R. Srimath Kandana, S. M. Zakeeruddin, A. Petrozza, A. Abate, M. K. Nazeeruddin, M. Grätzel, and A. Hagfeldt, Energy Environ. Sci., 8, 2928 (2015).

10. M. M. Lee, J. Teuscher, T. Miyasaka, T. N. Murakami, and H. J. Snaith, Science, 338, 643 (2012).

11. J. Burschka, N. Pellet, S.-J. Moon, R. Humphry-Baker, P. Gao, M. K. Nazeeruddin, and M. Grätzel, Nature, 499, 316 (2013).

12. K. Liang, D. B. Mitzi, and M. T. Prikas, Chem. Mater, 10, 403 (1998).

13. G. Niu, X. Guo, and L. Wang, J. Mater. Chem. A, 3, 8970 (2015).

14. J. J. Gutierrez-Sevillano, S. Ahmad, S. Calero, and J. A. Anta, Phys. Chem. Chem. Phys., 17, 22770 (2015)

15. N. J. Jeon, J. H. Noh, Y. C. Kim, W. S. Yang, S. Ryu, and S. I. Seok, Nat. Mater, 13 897 (2014).

16. Y. Wang, X. Li, J. Song, L. Xiao, H. Zeng, and H. Sun, Adv. Mater, 27, 7101 (2015).

17. L. Protesescu, S. Yakunin, M. I. Bodnarchuk, F. Krieg, R. Caputo, C. H. Hedon, R. X. Yang, A. Walsh, and M. V. Kovalenko, Nano Lett., 15, 3692 (2015).

18. W. Q. Hong, J. Phys. D:Appl. Phys., 22, 1384 (1989).

19. T. J. Jacobsson, M. Pazoki, A. Hagfeldt, and T. Edvinsson, J. Phys. Chem. C, 119, 25673 (2015).

20. M. Baibarac, N. Preda, L. Mihut, I. Baltog, S. Lefrant, and J. Y. Mevellec, J Phys.: Condens. Matter, 16, 2345 (2004).

21. G. Zhu, P. Liu, M. Hojamberdiev, J.-P. Zhou, X. Huang, B. Feng, and R. Yang, Appl. Phys. A., 98, 299 (2010).

22. T. Baikie, Y. Fang, J. M. Kadro, M. Schreyer, F. Wei, S. G. Mhaisalkar, M. Graetzel, and T. J. White, J. Mater. Chem. A, 1, 5628 (2013).

23. T. J. Jacobsson, L. J. Schwan, M. Ottosson, A. Hagfeldt, and T. Edvinsson, Inorg Chem., 54, 10678 (2015).

24. I. D. Baikie, A. C. Grain, J. Sutherland, and J. Law, Appl. Surf. Sc., 323, 45 (2014).

25. Y. Dang, Y. Liu, Y. Sun, D. Yuan, X. Liu, W. Lu, G. Liu, H. Xia, and X. Tao, Cryst. Eng. Comm., 17, 665 (2015).

26. Z. Zheng, A. Liu, S. Wang, Y. Wang, Z. Li, W. M. Lau, and L. Zhang, J. Mater. Chem., 15, 4555 (2005).

27. D. H. Cao, C. C. Stoumpos, O. K. Farha, J. T. Hupp, and M. G. Kanatzidis, J. Am. Chem. Soc., 137, 7843 (2015).

28. D. Shi, V. Adinolfi, R. Comin, M. Yuan, E. Alarousu, A. Buin, Y. Chen, S. Hoogland, A. Rothenberger, K. Katsiev, Y. Losovyj, X. Zhang, P. A. Dowben, O. F. Mohammed, E. H. Sargent, and O. M. Bakr, Sci. Mag., 347, 519 (2015).

29. J. A. Christians, J. S. Manser, and P. V. Kamat, J. Phys. Chem. Lett., 6, 2086 (2015)

30. J. J. Zhao, P. Wang, Z. H. Liu, L. Y. Wei, Z. Yang, H. R. Chen, X. Q. Fang, X. L. Liu, and Y. H. Mai, Dalton Trans., 44, 17841 (2015).

31. M. B. Johansson, B. Zietz, G. A. Niklasson, and L. Österlund, J. Appl. Phys., 115 (2014).

32. S. De Wolf, J. Holovsky, S.-J. Moon, P. Löper, B. Niesen, M. Ledinsky, F.-J. Haug, J.-H. Yum, and C. Ballif, J. Phys. Chem. Lett., 5, 1035 (2014).

33. F. Urbach, Phys. Rev. , 92, 1324 (1953).

34. G. C. Papavassiliou, G. Pagona, N. Karousis, G. A. Mousdis, I. Koutselas, and A. Vassilakopoulou, J. Mater. Chem., 22, 8271 (2012).

35. Q. Dong, Z. Xiao, Q. Wang, C. Bi, Y. Shao, and J. Huang, Solar and Alternative Energy, July 25 (2014).

36. C. Quarti, G. Grancini, E. Mosconi, P. Bruno, J. M. Ball, M. M. Lee, H. J. Snaith, M. Petrozza, and F. De Angelis, J. Phys. Chem. Lett., 5, 279 (2014).

37. B.-W. Park, S. M. Jain, X. Zhang, A. Hagfeldt, G. Boschloo, and T. Edvinsson, Acs. Nano, 9, 2088 (2015). 